Article

\title{
Research on Dual-Phase Non-Salient Pole Receiver for EV Dynamic Wireless Power Transfer System
}

\author{
Fandan Zhao $\mathbb{D}^{\mathrm{D}}$, Jinhai Jiang *(D), Shumei Cui, Chunbo Zhu and C. C. Chan \\ School of Electrical Engineering \& Automation, Harbin Institute of Technology, Harbin 150001, China; \\ zhaofandan_hit@126.com (F.Z.); cuism@hit.edu.cn (S.C.); zhuchunbo@hit.edu.cn (C.Z.); \\ ccchan@eee.hku.hk (C.C.C.) \\ * Correspondence: jiangjinhai@hit.edu.cn; Tel.: +86-185-0460-5367
}

Citation: Zhao, F.; Jiang, J.; Cui, S.; Zhu, C.; Chan, C.C. Research on Dual-Phase Non-Salient Pole Receiver for EV Dynamic Wireless Power Transfer System. World Electr. Veh. J. 2021, 12, 157. https://doi.org/ 10.3390/wevj12030157

Academic Editor: Peter Van den Bossche

Received: 23 August 2021 Accepted: 15 September 2021 Published: 19 September 2021

Publisher's Note: MDPI stays neutral with regard to jurisdictional claims in published maps and institutional affiliations.

Copyright: (c) 2021 by the authors. Licensee MDPI, Basel, Switzerland. This article is an open access article distributed under the terms and conditions of the Creative Commons Attribution (CC BY) license (https:// creativecommons.org/licenses/by/ $4.0 /)$.

\begin{abstract}
Dynamic wireless power transfer (DWPT) technology shows a vast development prospect for EV application, with advantages of reducing the demand for battery capacity and improving the user experience. However, the need to improve output performance leads to a challenge in receiver design with limited space and allowable load on the EV side. In this paper, a design of a dual-phase non-salient pole (NSP) receiver for the EV DWPT system with bipolar transmitter is proposed, aiming at providing a solution to the contradiction between reducing the volume or cost and improving the misalignment tolerance of the receiver. The coupling principle of the proposed receiver is analyzed. The structure parameters are optimized by the finite-element simulation method. Combined with specific design indexes, it is proven by comparison with the existing dual-phase receiver that the proposed receiver is $35.4 \%$ smaller in volume and needs $47.0 \%$ shorter wires. Moreover, the significant advantage of the proposed dual-phase NSP receiver in misalignment tolerance is verified by simulations and experimental comparisons.
\end{abstract}

Keywords: bipolar; dynamic charging; EV (electric vehicle); finite element calculation; wireless charging

\section{Introduction}

Dynamic wireless power transfer (DWPT) technology is a kind of non-contact power supply method to moving receivers. It shows a vast development prospect because it reduces the demand for battery capacity and improves the user experience [1-3].

Magnetic coupler, which is basically classified into long-rail type [4-6], coil array type [7,8] and bipolar type [9-11], is a key component of a DWPT system [12,13]. The bipolar type has good application prospect because it reduces the difficulty of deployment and cost by using a narrow transmitter to realize the transmission of a large amount of power [9]. However, the disadvantage is that the need to compensate the weak-coupling area and improve misalignment tolerance leads to the larger size and higher cost of the receiver [14]. In order to solve the problem, a dual-phase non-salient pole (NSP) receiver is proposed in this paper.

The name of non-salient pole comes from motor theory and is opposite to salient pole (SP). The typical SP structure [15] consists of a pole shoe, pole body and yoke. Each winding is wound around the pole body and produces a magnetic pole. In contrast, a pole shoe and pole body are not needed in the typical NSP structure [16]. The winding is wound around the yoke and produces a pair of opposite magnetic poles. According to the analysis of the relationship between winding deployment and magnetic pole distribution, the introduced bipolar type can be classified into SP and NSP forms. Therefore, the existing I-type [9], S-type [10] and N-type [11] transmitters belong to SP form and DD type [17], sandwich type [11] or DD-OQO type [18,19] receivers, which match the transmitter and can also be regarded as SP form with the special case of an extremely thin pole shoe and pole body. 
In this paper, the coupling principle of the proposed dual-phase NSP receiver is analyzed by a magnetic circuit. The relationship between structure parameters and coupling characteristics is discussed, and the performance is compared by a finite-element simulation and an experiment under the same design indexes.

\section{Analysis of Basic Principle}

The overall configuration of the dual-phase NSP receiver is shown in Figure 1. The design of the transmitter is the same as that in [18], ensuring fair comparison with the designed dual-phase NSP receiver and the existing dual-phase DD-OQO receiver. The dual-phase NSP receiver consists of two of the same modules and a shielding plate above the modules. In a module, $\mathrm{Q}$ winding consists of two sub windings with opposite winding directions. The connection of the dual-phase NSP receiver in the system is shown in Figure $1 \mathrm{~b}$. All $\mathrm{D}$ windings are in series to the $\mathrm{D}$-phase receiver and all $\mathrm{Q}$ windings are in series to the Q-phase receiver.

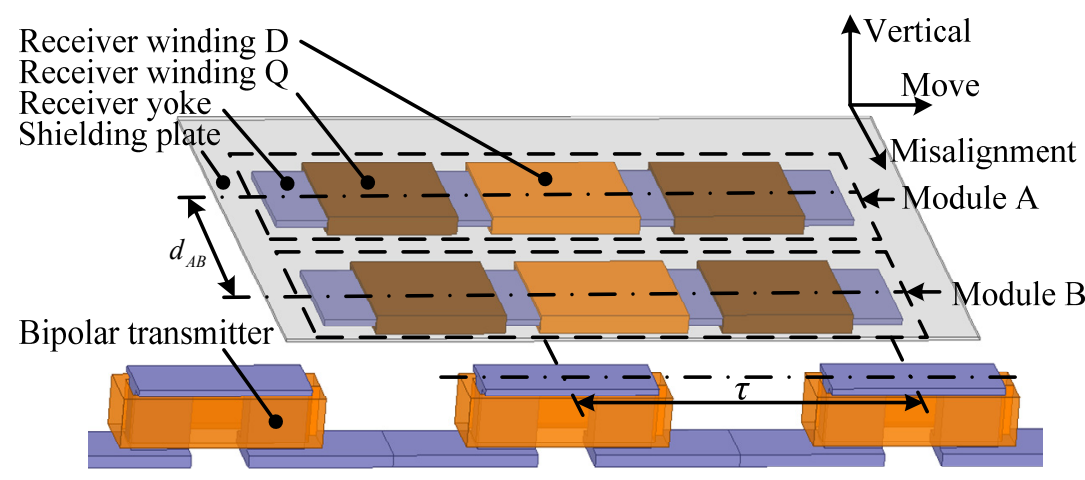

(a)

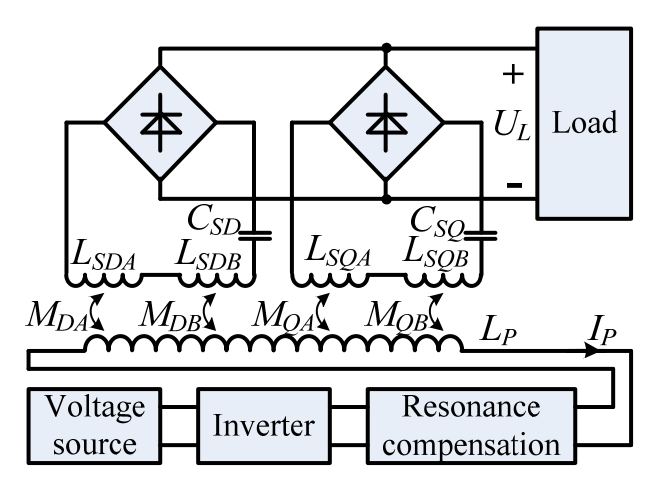

(b)

Figure 1. Overall configuration of the dual-phase NSP receiver: (a) Structure of the magnetic coupler with the dual-phase NSP receiver; (b) Connection of the dual-phase NSP receiver in the system.

When moving along the bipolar transmitter, the dual-phase NSP receiver varies between two modes, as shown in Figure 2. In Mode 1, the coupling is stronger in the D-phase receiver. In Mode 2, the coupling is stronger in the Q-phase receiver. According to the system in Figure 1b, the load voltage with moving distance $x$ is calculated by:

$$
\begin{aligned}
U_{L}(x) & =\omega_{0} I_{P} k_{d c-a c} \max \left(M_{D A}(x)+M_{D B}(x), M_{D A}(x)+M_{D B}(x)\right) \\
& =\frac{\pi}{2 \sqrt{2}} \omega_{0} I_{P} \max \left(M_{D A}(x)+M_{D B}(x), M_{D A}(x)+M_{D B}(x)\right)
\end{aligned}
$$

where $\omega_{0}$ is the resonant frequency, $I_{P}$ is RMS current of the transmitter and $k_{d c-a c}$ is the gain from the average voltage of the DC side to the RMS voltage of the AC side [20].

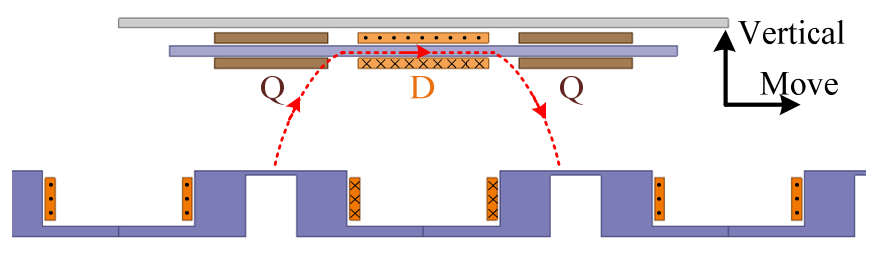

(a)

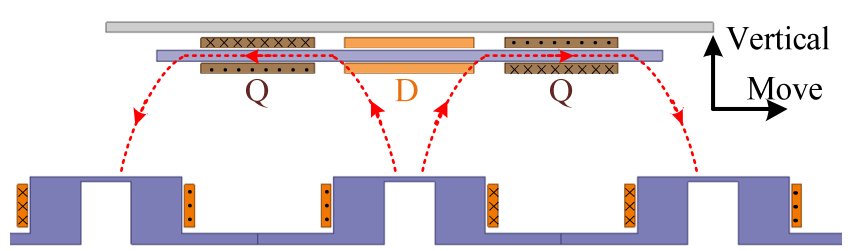

(b)

Figure 2. Two modes of the dual-phase NSP receiver along move direction: (a) Mode 1; (b) Mode 2.

The characteristic of mutual inductance of the dual-phase NSP receiver with moving distance is shown in Figure 3a. $\tau$ is the center distance between the adjacent magnetic poles in the transmitter. The weak coupling area is compensated and the fluctuation factor [12] is 
reduced. In addition, it is obvious that D-phase and the Q-phase receivers are decoupled to avoid nonideal power reduction.

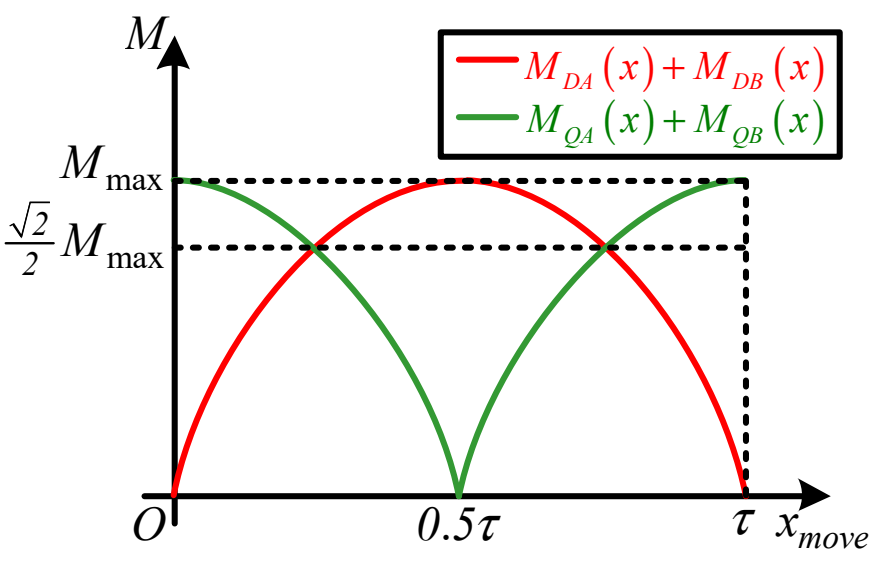

(a)

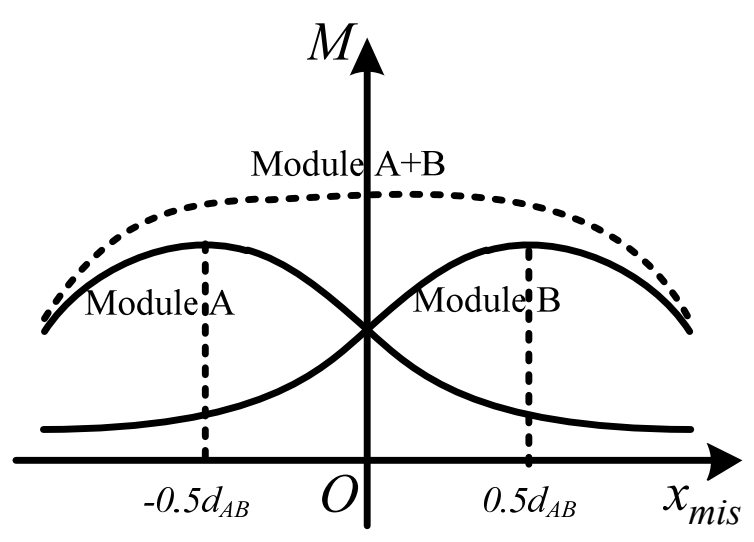

(b)

Figure 3. The characteristic of mutual inductance of the dual-phase NSP receiver with (a) moving distance and (b) misalignment distance.

The misalignment tolerance of a receiver varies with moving distance, and it is weakest in the exchange position of Mode 1 and Mode 2. The characteristic of mutual inductance of the dual-phase NSP receiver with misalignment is shown in Figure $3 \mathbf{b}$, where $d_{A B}$ is the center distance between two modules. Two modules compensate each other and reduce the sensitivity of the mutual inductance to the misalignment. It is possible for mutual inductance to be unchanged within a certain range of misalignment through optimized design.

\section{Parameter Optimization Analysis}

The main structure parameters of a dual-phase NSP receiver is shown in Figure 4, and a design case is given in Table 1 to provide a reference point for analyzing the influence of structure parameters on the performance of a dual-phase NSP receiver by finite-element simulation. In order to simplify the analysis, parameters such as wire diameter, winding width, magnetic core thickness and distance between module and shielding plate are not discussed, but the values of these parameters are ensured to meet the design requirements.

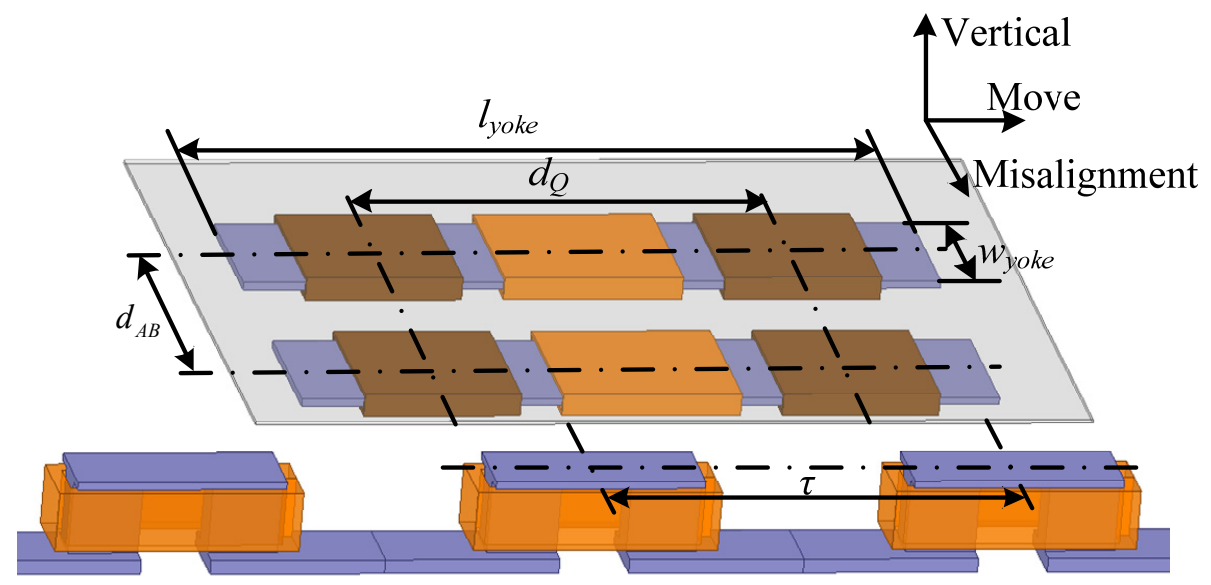

Figure 4. The main structure parameters of the dual-phase NSP receiver. 
Table 1. The design case of the dual-phase NSP receiver.

\begin{tabular}{lcc}
\hline \multicolumn{1}{c}{ Structure Parameter } & Symbol & Value \\
\hline Center distance between adjacent magnetic poles & $\tau$ & $600 \mathrm{~mm}$ \\
Length of receiver yoke & $l_{\text {yoke }}$ & $1000 \mathrm{~mm}$ \\
Width of receiver yoke & $w_{\text {yoke }}$ & $200 \mathrm{~mm}$ \\
Center distance of receiver winding Q & $d_{Q}$ & $600 \mathrm{~mm}$ \\
Center distance between receiver module A and B & $d_{A B}$ & $400 \mathrm{~mm}$ \\
\hline
\end{tabular}

\subsection{Length and Width of the Receiver Yoke}

Figure 5 shows the mutual inductance and the coupling coefficient of single-turn winding $\mathrm{D}$ in Mode 1 and single-turn winding $\mathrm{Q}$ in Mode 2 with different lengths of receiver yoke $l_{\text {yoke }}$ and widths of receiver yoke $w_{\text {yoke }}$. Obviously, a larger $w_{\text {yoke }}$ leads to a larger end face of the coupling flux, and then, the mutual inductance and the coupling coefficient are increased. However, the influence is weakened along with a larger $w_{\text {yoke }}$. In Figure $5 \mathrm{a}, l_{\text {yoke }}$ has little effect on the mutual inductance of winding D; due to that, a pair of adjacent magnetic poles is mostly covered by the receiver yoke in Mode 1. However, the coupling coefficient decreases in Figure $5 \mathrm{~b}$ with too large $l_{\text {yoke }}$ because it provies a leakage flux path for other magnetic poles of the transmitter. A winging $\mathrm{Q}$ consists of two sub windings, and the mutual inductance of each sub winding is weaker because of smaller covered area of adjacent magnetic poles by the receiver yoke in Mode 2. This also means that $l_{\text {yoke }}$ has a larger effect on the mutual inductance and the coupling coefficient, which are shown in Figure $5 \mathrm{c}$,d. In summary, under the spacing limitation of the receiver in engineering design, the receiver yoke is preferred to be longer and narrower, to significantly improve the mutual inductance of winding $Q$ with less sacrifice of winding $D$ and to reduce the wire consumption under the same design requirements.

\subsection{Center Distance of Receiver Winding $Q$}

It is revealed in Figure 2 that adjusting the center distance of receiver winding $\mathrm{Q} d_{Q}$ has no effect on the coupling mode of the dual-phase NSP receiver. To optimized $d_{Q}$, the inner magnetic flux of the position in the receiver yoke surrounded by the winding $Q$ should be larger when the magnetic flux is generated by only one transmitter. It is shown in Figure 6 that the optimized $d_{Q}$ is close to the center distance between adjacent magnetic poles $\tau$ of transmitter. It should be noticed that when $d_{Q}$ is $600 \mathrm{~mm}$, the mutual inductance is less effected by $\tau$, which shows possible interoperability between the dual-phase NSP receiver and different designs of the transmitter.

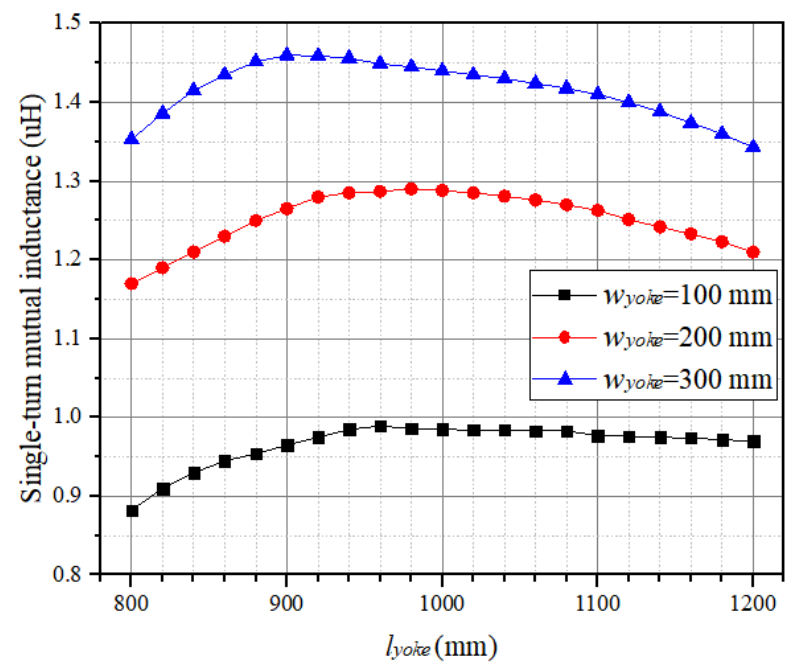

(a)

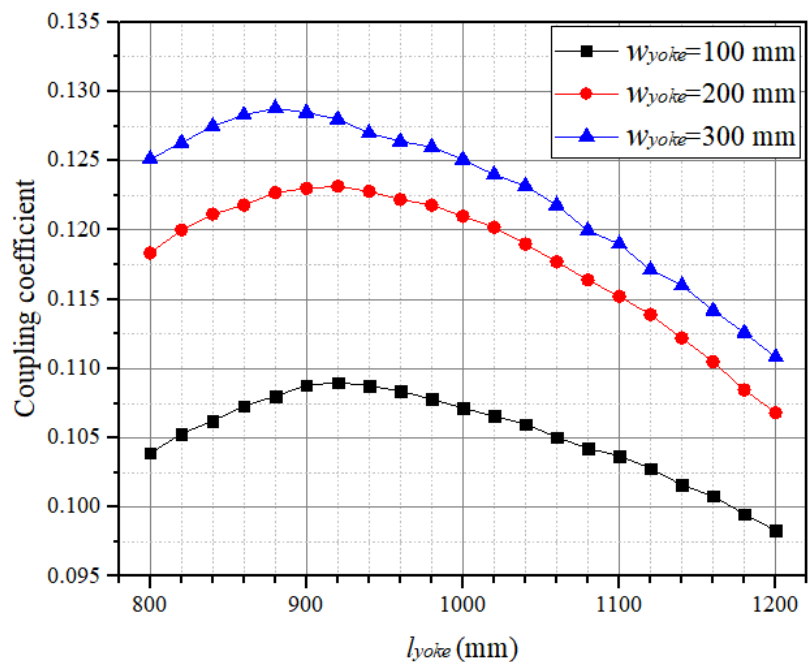

(b)

Figure 5. Cont. 


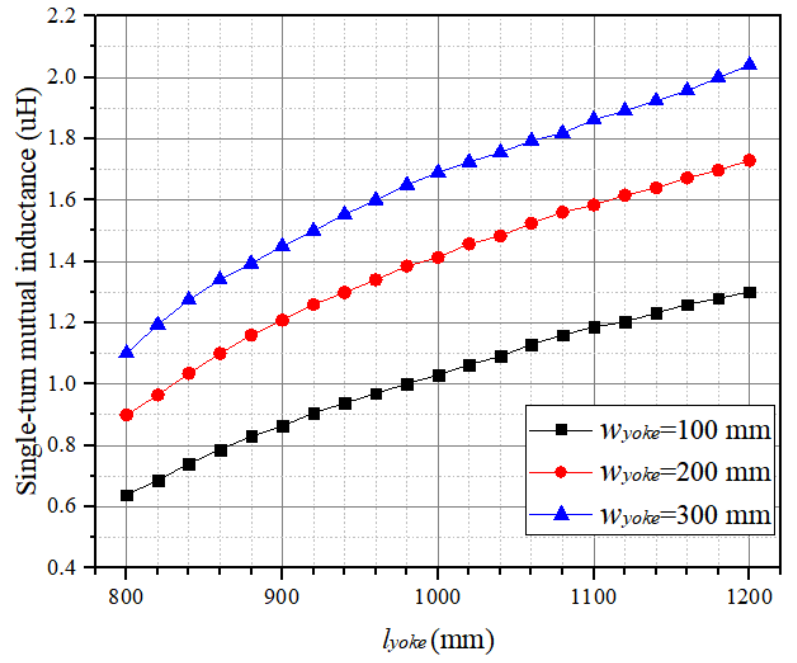

(c)

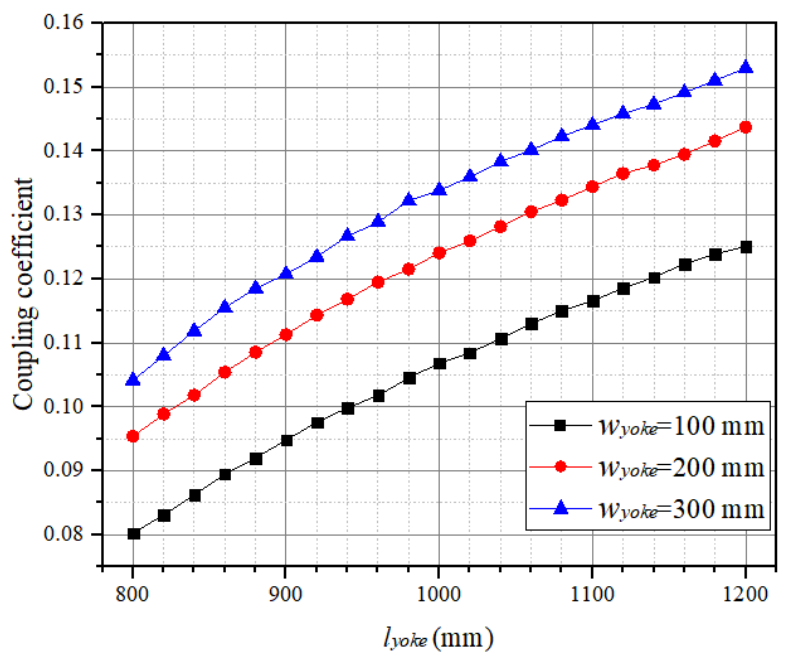

(d)

Figure 5. (a) The mutual inductance and (b) the coupling coefficient of single-turn winding D in Mode 1, and (c) the mutual inductance and (d) the coupling coefficient of single-turn winding $\mathrm{Q}$ in Mode 2.

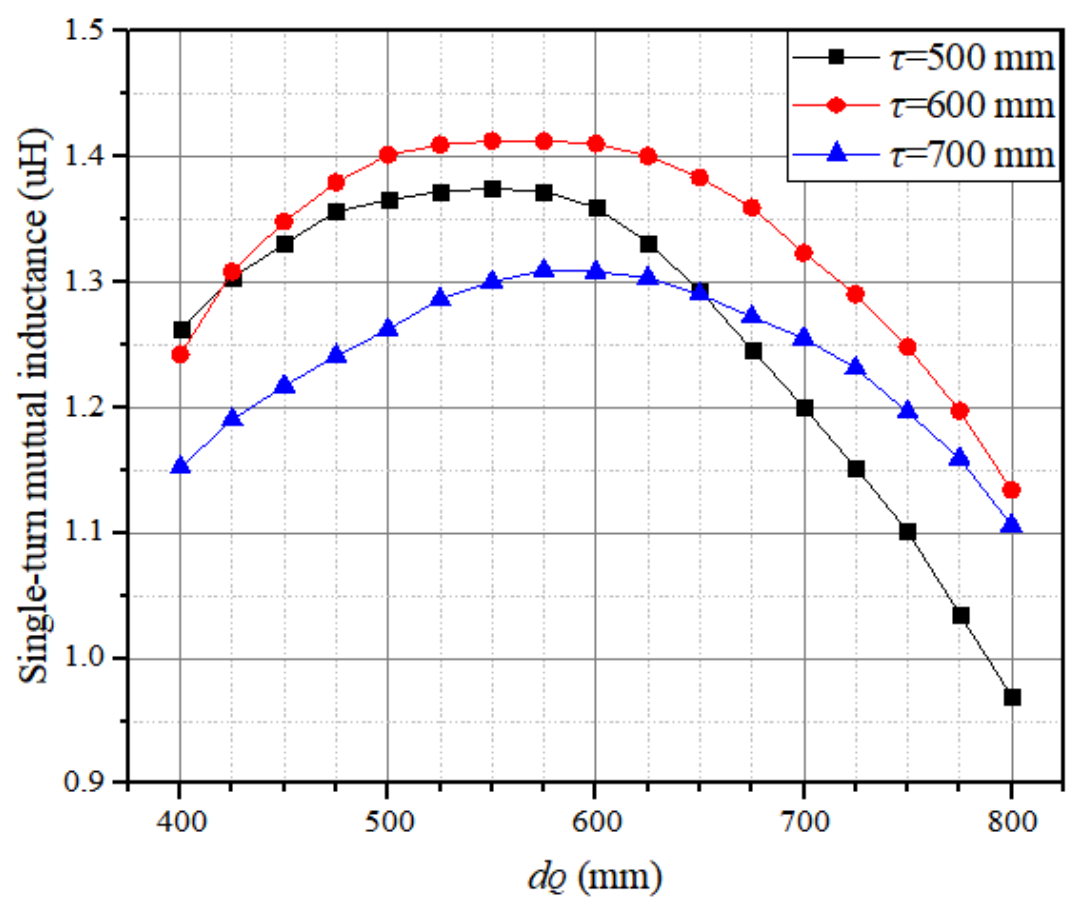

Figure 6. The single-turn mutual inductance of winding $Q$ with different $d_{Q}$ and $\tau$ values.

\subsection{Center Distance between Receiver Module A and B}

The characteristic of normalized mutual inductance in misalignment with different center distances between receiver module $\mathrm{A}$ and $\mathrm{B} d_{A B}$ is shown in Figure 7. According to the principal analysis of coupling compensation by two modules in Figure $3 b$, it is shown that when $d_{A B}$ is smaller than $400 \mathrm{~mm}$, it is in a compensation state, when $d_{A B}$ is equal to $400 \mathrm{~mm}$, it is in a critical compensation state, and when $d_{A B}$ is larger than $400 \mathrm{~mm}$, it is in an overcompensation state. In the critical compensation state, the mutual inductance is unchanged within $160 \mathrm{~mm}$ of misalignment, which is quite valuable for improving misalignment tolerance. 


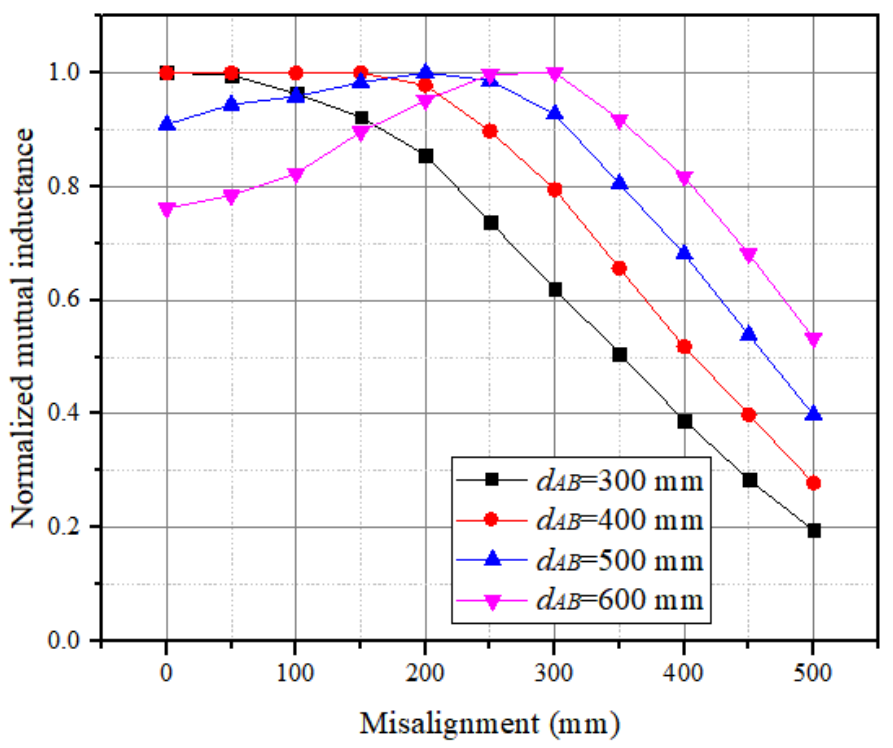

Figure 7. The normalized mutual inductance in misalignment with different $d_{A B}$ values.

\section{Design and Simulation Verification}

To evaluate the performance and the practicability of the dual-phase NSP receiver, a dual-phase DD-OQO receiver [18] shown in Figure 8, which is the latest structure of the dual-phase receiver, is considered as reference. These two receivers are designed by the finite-element simulation method. Design indexes and the information of the design results are shown in Table 2. In the comparison, the length and the width of the shielding plate in the dual-phase NSP receiver are the same as those of DD-OQO receiver, indicating same area needed under the chassis of a vehicle. However, the module size and the overall size of the dual-phase NSP receiver are significantly smaller than those of the dual-phase DD-OQO receiver. Using dual-phase, the NSP receiver can reduce $35.4 \%$ of the occupied volume and $47.0 \%$ of the wire consumption, which also indicates lighter weight of the receiver.

To compare the misalignment tolerance of these two receivers, the normalized receiving voltage with different moving distances and misalignments is shown in Figure 9. It is obtained that the higher the allowable lower limit of the normalized receiving voltage is, the more obvious advantage the dual-phase NSP receiver will have. For example, the weakest misalignment tolerance of the dual-phase NSP receiver is about $10 \%$ larger when the normalized allowable lower limit is 0.5 and about $67 \%$ larger when the normalized allowable lower limit is 0.7 .

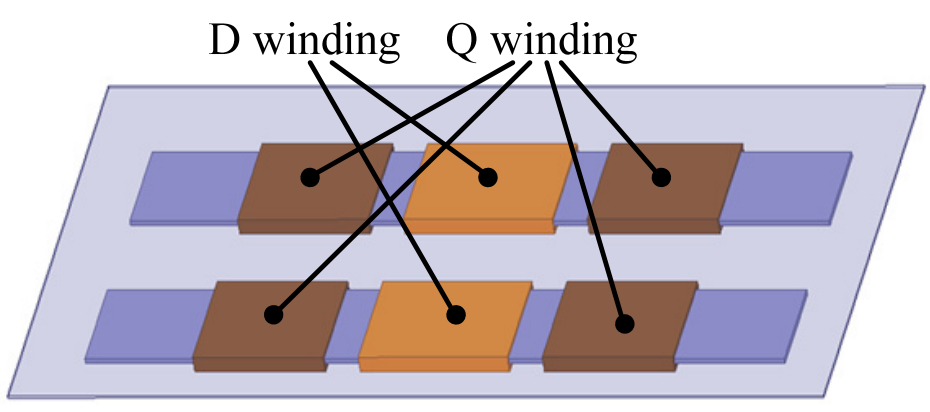

(a)

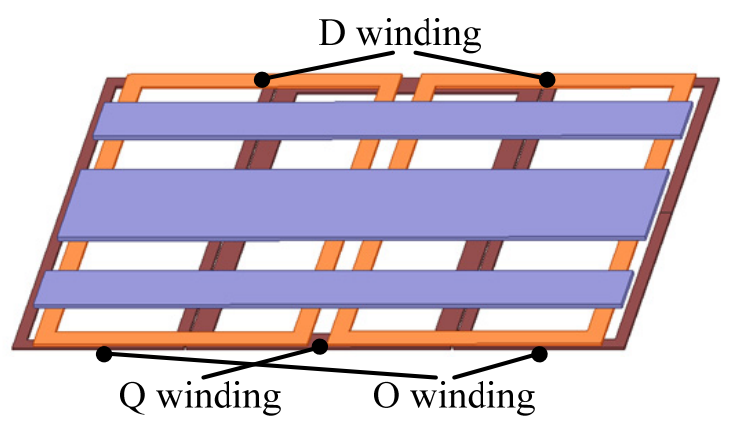

(b)

Figure 8. Diagrams of (a) the proposed dual-phase NSP receiver and (b) the reference dual-phase DD-OQO receiver. 
Table 2. Design indexes and the information of the design results.

\begin{tabular}{ccc}
\hline Type of Receiver & Dual-Phase NSP & Dual-Phase DD-OQO \\
\hline Resonant frequency & & $20 \mathrm{kHz}$ \\
Transmission distance & & $700 \mathrm{~mm}$ \\
Rated transmitter current & & $0.1 \mathrm{~mm} \times 2500$ strands \\
Specification of Litz wire & PC95 \\
Material of magnetic cores & $1200 \mathrm{~mm} \times 800 \mathrm{~mm}$ & $1200 \mathrm{~mm} \times 800 \mathrm{~mm}$ \\
Overall area (including shielding) & $(1 \mathrm{module})$ & $1200 \mathrm{~mm} \times 800 \mathrm{~mm} \times 30 \mathrm{~mm}$ \\
Size (except shielding) & $620 \mathrm{~mm}$ & $800 \mathrm{~mm}$ \\
Width of the receiver (except shielding) & D winding: 16 turns & D winding: 6 turns \\
Number of turns & Q winding: 14 turns & O winding: 4 turns \\
Length of wires & $36.96 \mathrm{~m}$ & $69.80 \mathrm{~m}$ \\
Volume of magnetic cores & $4000 \mathrm{~cm}^{3}$ & $4800 \mathrm{~cm}{ }^{3}$
\end{tabular}

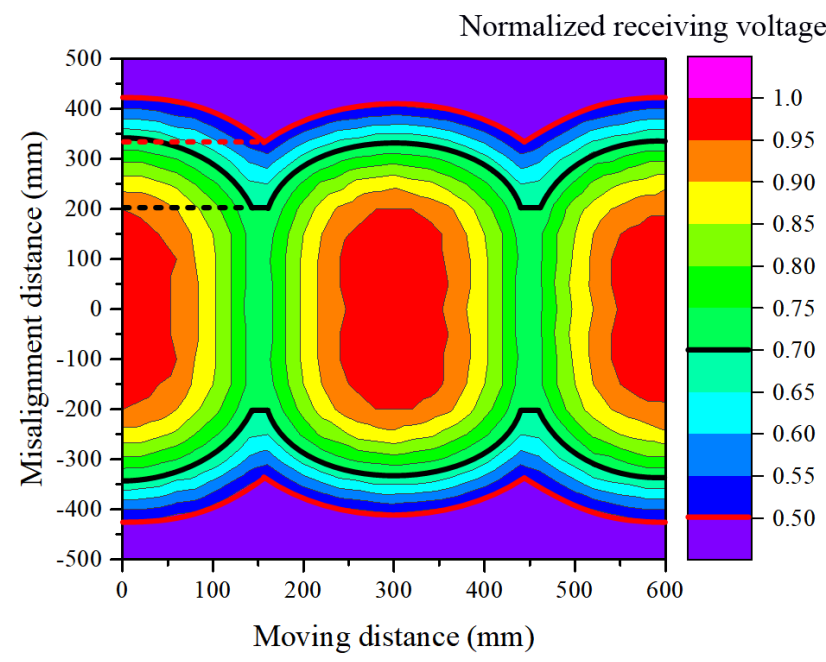

(a)

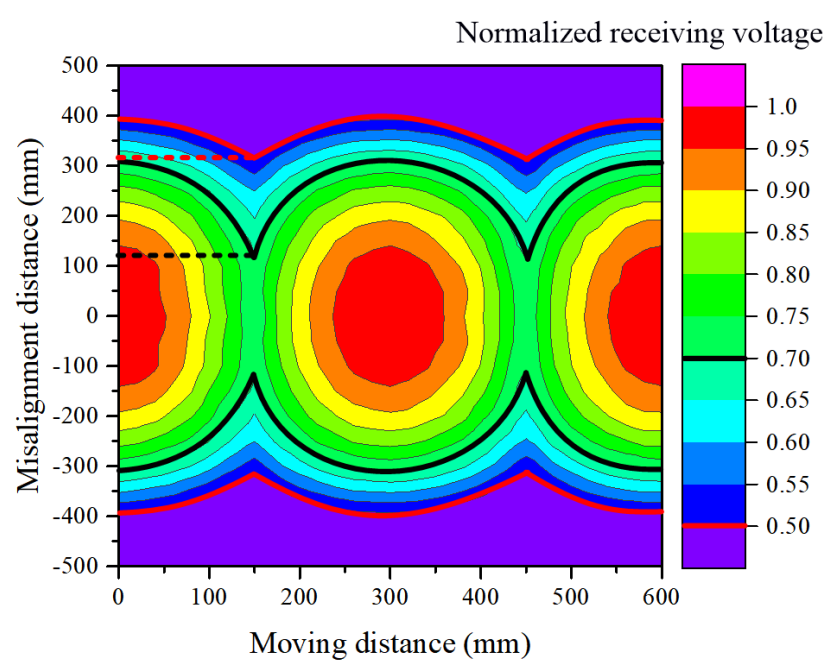

(b)

Figure 9. Simulated normalized receiving voltage of (a) the proposed dual-phase NSP receiver and (b) the reference dual-phase DD-OQO receiver with moving distance and misalignment distance.

\section{Experiment}

The experimental system used to compare the dual-phase NSP receiver and the dual-phase DD-OQO receiver is shown in Figure 10. The experimental conditions of the two receivers are the same, and the experimental parameters are consistent with Table 2. Figure 11 shows the details of two receivers, which are made according to the design dimensions in Table 2. Measured parameters of the magnetic coupler with the dual-phase NSP receiver or the dual-phase DD-OQO receiver are shown in Table 3. The maximum mutual inductance of the two receivers is close, indicating that the power output capacity of the two receivers is similar under the same experimental conditions. In Table 2, the wire consumption of the dual-phase NSP receiver is significantly shorter than that of the DD-OQO receiver. However, the AC internal resistance of the dual-phase NSP receiver is not correspondingly smaller in the measure result. It is implied that the structure of the receiver has a non-ignorable influence on the $\mathrm{AC}$ internal resistance characteristics of the winding. 


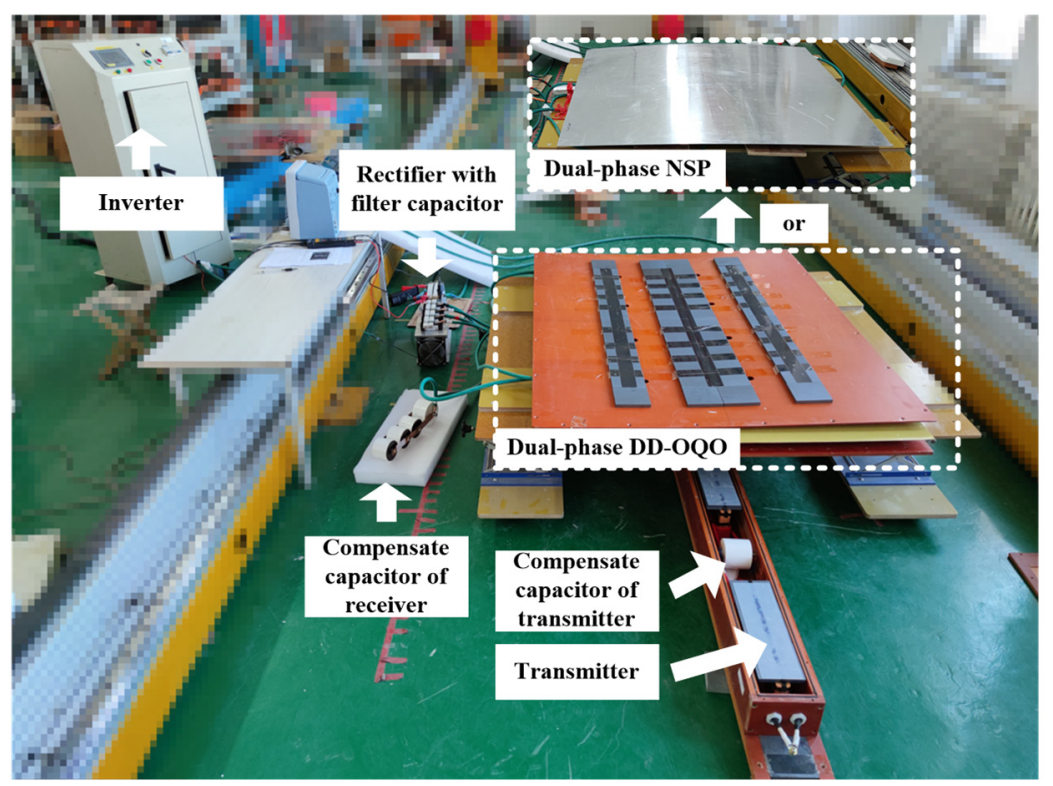

Figure 10. Experimental system for comparing the dual-phase NSP receiver and the dual-phase DD-OQO receiver.

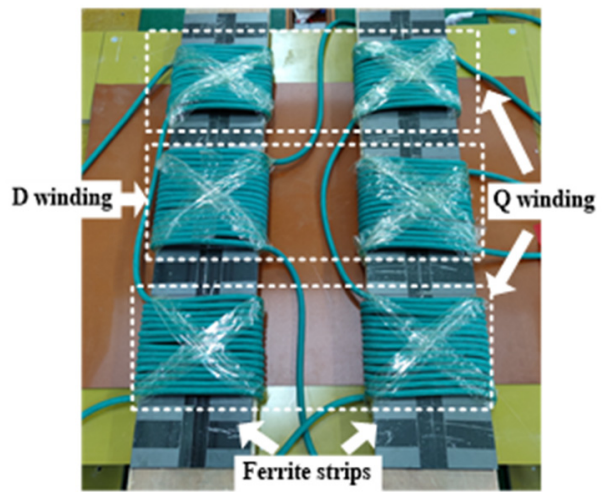

(a)

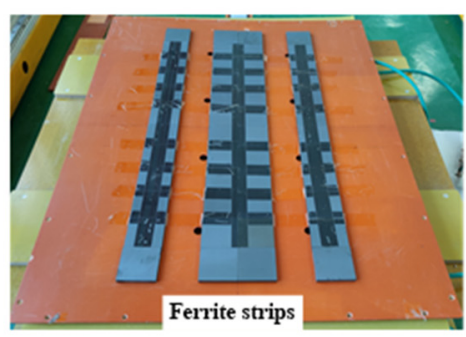

Ferrite strips
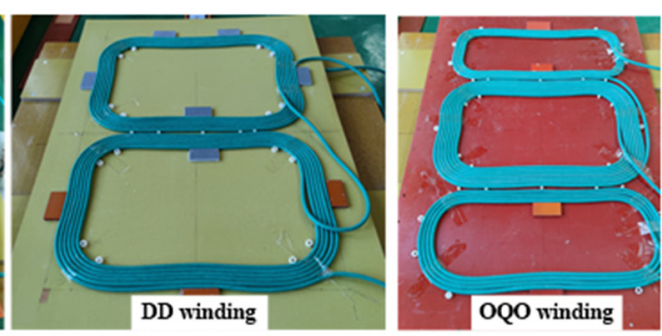

(b)

Figure 11. Details of two receivers: (a) Dual-phase NSP receiver; (b) Dual-phase DD-OQO receiver.

Table 3. Measured parameters of the magnetic coupler.

\begin{tabular}{ccc}
\hline Parameter & Symbol & Value \\
\hline Inductance of transmitter & $L_{P}$ & $210 \mu \mathrm{H}$ \\
Inductance of dual-phase NSP receiver & $L_{S D A}+L_{S D B}$ & $236.0 \mu \mathrm{H}$ \\
& $L_{S Q A}+L_{S Q B}$ & $262.2 \mu \mathrm{H}$ \\
Inductance of dual-phase DD-OQO receiver & $L_{S-D D}$ & $153.7 \mu \mathrm{H}$ \\
AC internal resistance of transmitter & $L_{S-O Q O}$ & $172.9 \mu \mathrm{H}$ \\
AC internal resistance of dual-phase NSP receiver & $r_{P}$ & $60 \mathrm{~m} \Omega$ \\
AC internal resistance of dual-phase DD-OQO receiver & $r_{S D A}+r_{S D B}$ & $63 \mathrm{~m} \Omega$ \\
Max mutual inducatance with dual-phase NSP receiver & $r_{S Q A}+r_{S Q B}$ & $78 \mathrm{~m} \Omega$ \\
Max mutual inducatance with dual-phase & $r_{S-O Q O}$ & $65 \mathrm{~m} \Omega$ \\
DD-OQO receiver & $M_{N S P}$ & $160.0 \mu \mathrm{m} \Omega$ \\
\hline
\end{tabular}

The position where the maximum mutual inductance is obtained is taken as the initial position of the receiver. In this position, power waveforms of the system with two receivers 
are shown in Figure 12. The calculated mutual inductance according to the load voltage and transmitter current in two systems are close to measured values in Table 3. In general, the system states with two receivers are near the same. In fact, the mutual inductance with the dual-phase DD-OQO receiver is slightly larger than that with the dual-phase NSP receiver, and thus, the output power with the dual-phase DD-OQO receiver is slightly larger correspondingly.

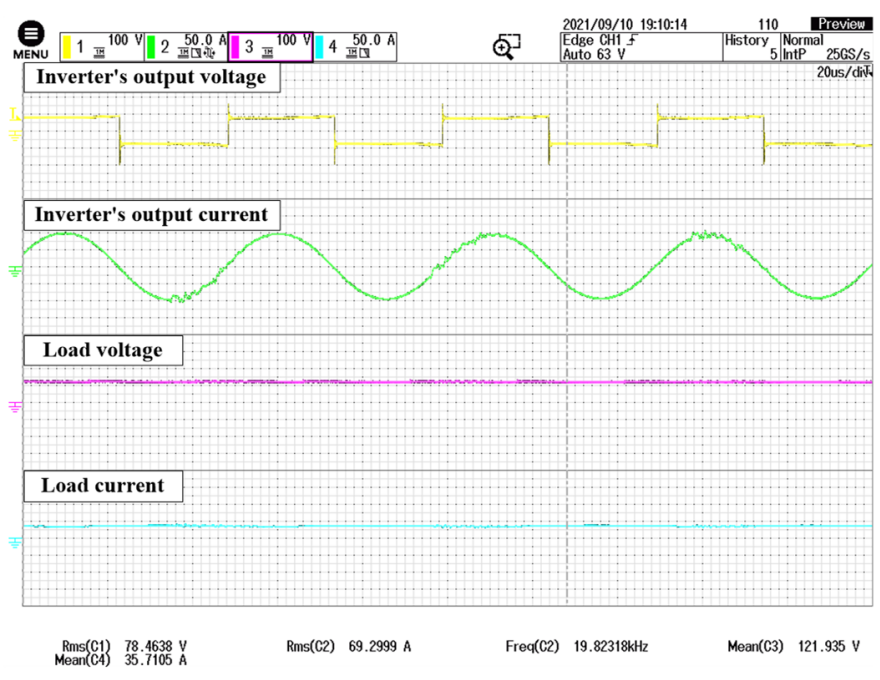

(a)

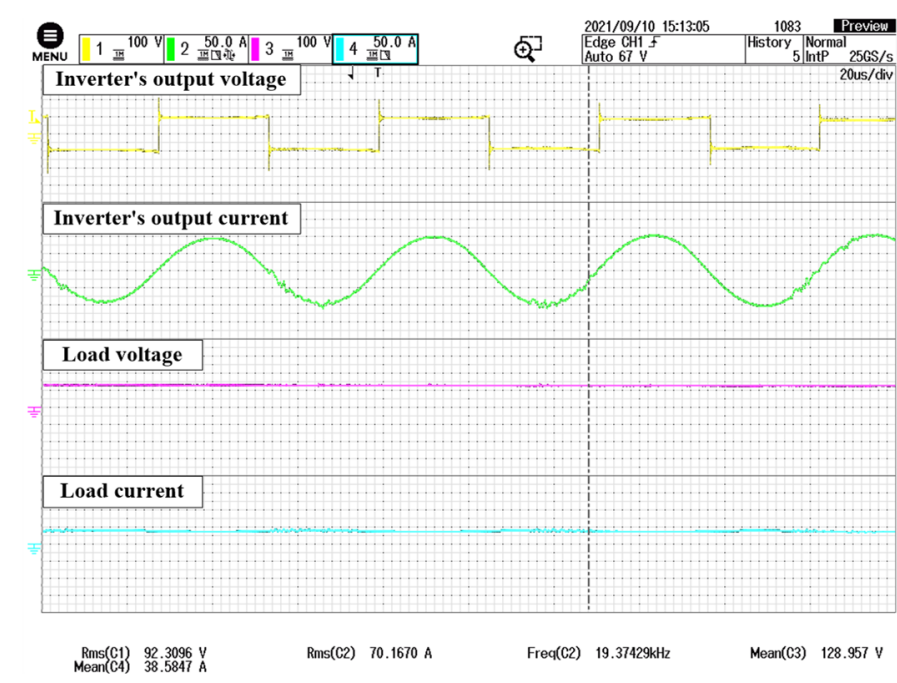

(b)

Figure 12. Power waveforms of the system with two receivers: (a) the dual-phase NSP receiver; (b) the dual-phase DD-OQO receiver.

In order to verify and compare the dynamic performance of the two receivers, the output characteristics of the two receivers under different moving distances and misalignments from the initial position are measured and shown in Figures 13 and 14. In the experiment, the load resistance is fixed. In Figure 13, the fluctuation characteristics of the output power of two receivers are consistent with the general dual-phase receiver. Since the mutual inductance of the dual-phase DD-OQO receiver is slightly larger than that of the dual-phase NSP receiver, the output power of the dual-phase DD-OQO receiver is a little larger correspondingly. Although the measured AC internal resistance of the dual-phase NSP receiver is close to that of the dual-phase DD-OQO receiver, the additional shielding loss of the dual-phase NSP receiver further reduces the efficiency of the dual-phase NSP receiver.

The output characteristics of two receivers with different misalignments from the maximum mutual inductance position and the minimum mutual inductance position are shown in Figure 14. The experimental results of the dual-phase NSP receiver are basically consistent with the simulation analysis. Obviously, the output stability of the dual-phase NSP receiver is better than that of the dual-phase DD-OQO receiver within $200 \mathrm{~mm}$ of misalignment. Combined with the discussion of different allowable lower limits of the received voltage in Figure 9, when the allowable lower limit is $70 \%$ of the maximum value, the weakest misalignment tolerance of the dual-phase NSP receiver is about $250 \mathrm{~mm}$, but that of the dual-phase DD-OQO receiver is about $127 \mathrm{~mm}$. When the allowable lower limit is $50 \%$ of the maximum value, the weakest misalignment tolerance of the dual-phase NSP receiver is approximately larger than $300 \mathrm{~mm}$, but that of the dual-phase DD-OQO receiver is approximately $264 \mathrm{~mm}$. 


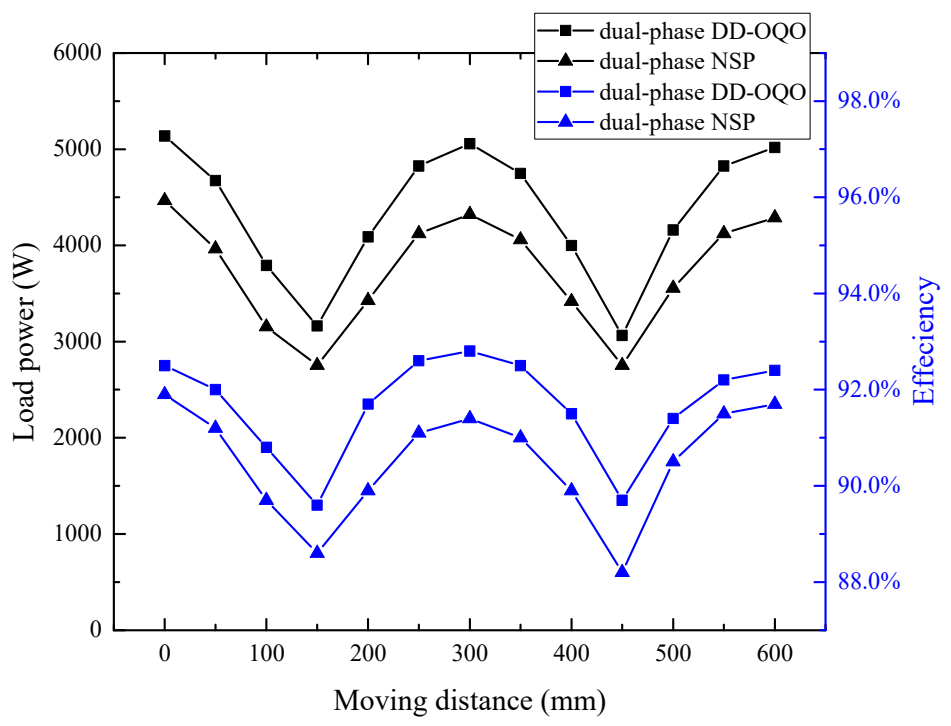

Figure 13. Output characteristics of the two receivers under different moving distances.

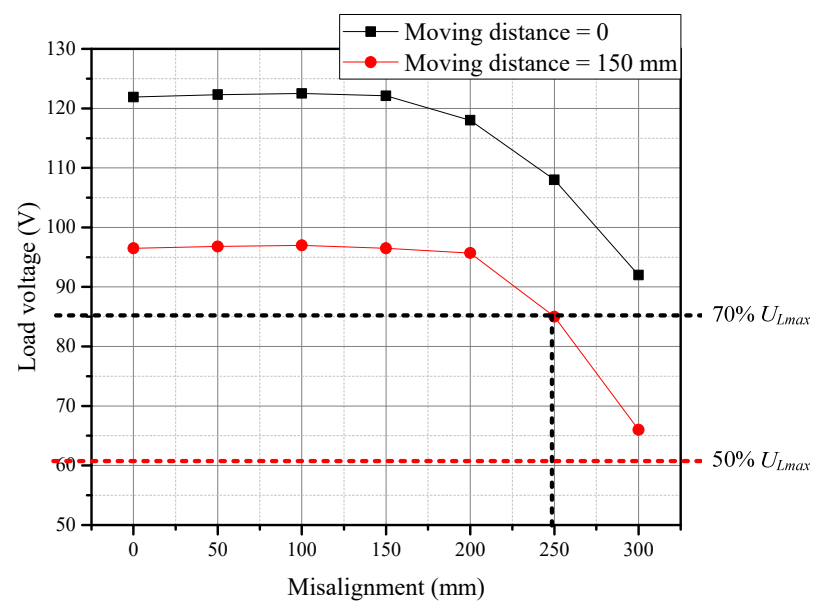

(a)

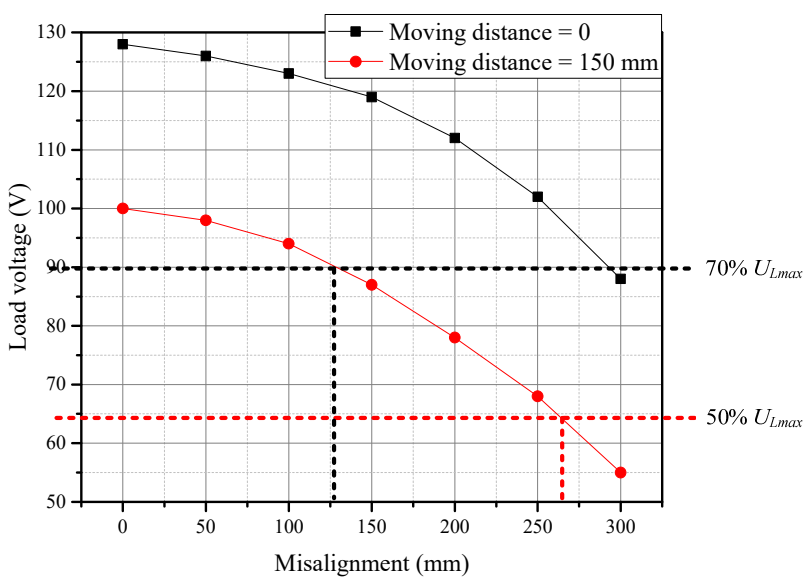

(b)

Figure 14. Output characteristics of two receivers with different misalignments from the maximum mutual inductance position and the minimum mutual inductance position: (a) the dual-phase NSP receiver; (b) the dual-phase DD-OQO receiver.

\section{Conclusions}

In this paper, the design of a dual-phase NSP receiver for an EV DWPT system with bipolar transmitter is proposed, compatible with existing dual-phase receivers, but with a smaller size, lower cost, and better misalignment tolerance. In essence, it reduces the fluctuation factor and decouples the two-phase windings. The sensitivity of mutual inductance to a certain misalignment is greatly reduced by using a dual-module structure. The advantages of the dual-phase NSP receiver are verified by comparing design, simulation and experiment results with the existing dual-phase DD-OQO receiver under the same design indexes. In the simulation results, the dual-phase NSP receiver is $35.4 \%$ smaller in volume, $47.0 \%$ shorter in wire consumption, and had a $10-67 \%$ improvement in misalignment tolerance according to different allowable lower limits of receiving voltage.

The effectiveness of the design and simulation results was verified by experimental comparison; however, an additional problem was found. Although the wire consumption of the dual-phase NSP receiver is significantly less, the AC internal resistance and total loss have no obvious advantage compared with the dual-phase DD-OQO receiver. Thus, further research will need to focus on the influence on $\mathrm{AC}$ internal resistance characteristics 
of the winding from the receiver structure, as well as the optimization of shielding loss of the dual-phase NSP receiver.

Author Contributions: Conceptualization, F.Z., J.J. and C.Z.; methodology, S.C. and C.C.C.; software, F.Z.; validation, J.J. and S.C.; formal analysis, C.Z.; investigation, F.Z.; resources, F.Z. and J.J.; data curation, J.J.; writing—original draft preparation, F.Z.; writing—review and editing, J.J.; visualization, F.Z.; supervision, J.J., S.C. and C.Z.; project administration, S.C. All authors have read and agreed to the published version of the manuscript.

Funding: This research was funded by the China Postdoctoral Science Foundation (Grant No. 2020M681094) and the National Natural Science Foundation of China (Grant No. 52007038).

Conflicts of Interest: The authors declare no conflict of interest.

\section{References}

1. Mi, C.C.; Buja, G.; Choi, S.Y.; Rim, C.T. Modern Advances in Wireless Power Transfer Systems for Roadway Powered Electric Vehicles. IEEE Trans. Ind. Electron. 2016, 63, 6533-6545. [CrossRef]

2. Machura, P.; de Santis, V.; Li, Q. Driving Range of Electric Vehicles Charged by Wireless Power Transfer. IEEE Trans. Veh. Technol. 2020, 69, 5968-5982. [CrossRef]

3. Fujita, T.; Yasuda, T.; Akagi, H. A Dynamic Wireless Power Transfer System Applicable to a Stationary System. IEEE Trans. Ind. Appl. 2017, 53, 3748-3757. [CrossRef]

4. Zaheer, A.; Budhia, M.; Kacprzak, D.; Covic, G.A. Magnetic Design of a 300 W Under-floor Contactless Power Transfer System. In Proceedings of the IECON 2011: 37th Annual Conference on IEEE Industrial Electronics Society, Melbourne, VIC, Australia, 7-10 November 2011.

5. Lee, S.; Huh, J.; Park, C.; Choi, N.; Cho, G.; Rim, C. On-Line Electric Vehicle using inductive power transfer system. In Proceedings of the 2010 IEEE Energy Conversion Congress and Exposition, Atlanta, GA, USA, 12-16 September 2010.

6. Wang, Y.; Lin, F.; Yang, Z.; Cai, P.; Liu, Z. Coils layout optimization of dynamic wireless power transfer system to realize output voltage stable. In Proceedings of the Power Electronics Conference (IPEC-Niigata 2018 -ECCE Asia) 2018 International, Niigata, Japan, 20-24 May 2018.

7. Miller, J.M.; Jones, P.T.; Li, J.; Onar, O.C. ORNL Experience and Challenges Facing Dynamic Wireless Power Charging of EV's. IEEE Circuits Syst. Mag. 2015, 15, 40-53. [CrossRef]

8. Xiang, L.; Li, X.; Tian, J.; Tian, Y. A Crossed DD Geometry and Its Double-Coil Excitation Method for Electric Vehicle Dynamic Wireless Charging Systems. Access IEEE 2018, 6, 45120-45128. [CrossRef]

9. Huh, J.; Lee, S.W.; Lee, W.Y.; Cho, G.H.; Rim, C.T. Narrow-Width Inductive Power Transfer System for Online Electrical Vehicles. IEEE Trans. Power Electron. 2011, 26, 3666-3679. [CrossRef]

10. Choi, S.Y.; Jeong, S.Y.; Gu, B.W.; Lim, G.C.; Rim, C.T. Ultraslim S-Type Power Supply Rails for Roadway-Powered Electric Vehicles Generalized Models on Self-Decoupled Dual Pick-Up Coils for a Large Lateral Tolerance. IEEE Trans. Power Electron. 2015, 30, 6456-6468. [CrossRef]

11. Wang, Z.; Cui, S.; Han, S.; Song, K.; Zhu, C.; Matveevich, M.I.; Yurievich, O.S. A Novel Magnetic Coupling Mechanism for Dynamic Wireless Charging System for Electric Vehicles. IEEE Trans. Veh. Technol. 2018, 67, 124-133. [CrossRef]

12. Boys, J.T.; Elliott, G.A.J.; Covic, G.A. An Appropriate Magnetic Coupling Co-Efficient for the Design and Comparison of ICPT Pickups. IEEE Trans. Power Electron. 2007, 22, 333-335. [CrossRef]

13. Zhang, X.; Yuan, Z.; Yang, Q.; Li, Y.; Zhu, J.; Li, Y. Coil Design and Efficiency Analysis for Dynamic Wireless Charging System for Electric Vehicles. IEEE Trans. Magn. 2016, 52, 1-4. [CrossRef]

14. Shin, J.; Shin, S.; Kim, Y.; Ahn, S.; Lee, S.; Jung, G.; Jeon, S.; Cho, D. Design and Implementation of Shaped Magnetic-ResonanceBased Wireless Power Transfer System for Roadway-Powered Moving Electric Vehicles. IEEE Trans. Ind. Electron. 2014, 61, 1179-1192. [CrossRef]

15. Shen, Y.; Lu, Q.; Huang, X. Analysis of a Novel Linear Doubly Salient Slot Permanent Magnet Motor. IEEE Trans. Magn. 2017, 53, 1-4. [CrossRef]

16. Lorilla, L.M.; Keim, T.A.; Lang, J.H.; Perreault, D.J. Topologies for future automotive generators. Part I. Modeling and analytics. In Proceedings of the 2005 IEEE Vehicle Power and Propulsion Conference, Chicago, IL, USA, 7 September 2005.

17. Cui, S.; Wang, Z.; Han, S.; Zhu, C.; Chan, C.C. Analysis and Design of Multiphase Receiver with Reduction of Output Fluctuation for EV Dynamic Wireless Charging System. IEEE Trans. Power Electron. 2019, 34, 4112-4124. [CrossRef]

18. Wang, Z.Y.; Zhang, J.T.; Huang, T.H.; Cui, S.M. Coil Design for High Coupling Performance for Two-phase Receiver of Dynamic Wireless Charging System. In Proceedings of the 2019 IEEE Wireless Power Transfer Conference (WPTC), London, UK, 18-21 June 2019.

19. Song, B.; Dong, S.; Li, Y.; Cui, S. A Dual-Layer Receiver with a Low Aspect Ratio and a Reduced Output Fluctuation for EV Dynamic Wireless Charging. IEEE Trans. Power Electron. 2020, 35, 10338-10351. [CrossRef]

20. Steigerwald, R.L. A comparison of half-bridge resonant converter topologies. IEEE Trans. Power Electron. 1988, 3, 174-182. [CrossRef] 